\title{
Experiences Teaching a Large Upper-Division Data Science Course Remotely
}

\author{
Suraj Rampure* \\ University of California, Berkeley \\ suraj.rampure@berkeley.edu
}

\author{
Allen Shen* \\ University of California, Berkeley \\ allenshen5@berkeley.edu
}

\author{
Josh Hug \\ University of California, Berkeley \\ hug@cs.berkeley.edu
}

\begin{abstract}
This summer, we introduced a number of structural changes to an existing upper-division data science course to optimize it for remote instruction. With hundreds of students situated in various time zones, a purely synchronous model was not feasible. Our course included a menu of synchronous and asynchronous activities, allowing students to consume content in the way they found most convenient and desirable.

We presented lectures asynchronously as a series of short prerecorded videos with conceptual questions following each video. We used the results of these conceptual questions to gauge students' preliminary understanding of the material and to identify common misconceptions. Each week ended with a single synchronous lecture recap session that clarified these misconceptions and provided a summary of the week's material. To supplement lecture content, we offered both prerecorded and live discussion sections, as well as live lab sections. Weekly surveys allowed us to adapt our course to address student concerns in real time.

Given the online nature of the course, we were able to depart from the orthodox paper-only exam format used by most courses at our institution. Instead, we held each of our three exams in a format optimized for its respective content. One midterm used a web tool that allowed for both multiple choice and free-response coding questions. The other midterm was on paper and required students to scan their exams. The final exam was a hybrid of the two formats.

In this experience report, we present the motivation, implementation details, and effectiveness of these changes.
\end{abstract}

\section{CCS CONCEPTS}

- Social and professional topics $\rightarrow$ Computing education; • Applied computing $\rightarrow$ E-learning.

\section{KEYWORDS}

Upper-division data science course; Asynchronous remote instruction; Synchronous remote instruction; Online exam formats

\section{ACM Reference Format:}

Suraj Rampure, Allen Shen, and Josh Hug. 2021. Experiences Teaching a Large Upper-Division Data Science Course Remotely. In Proceedings of the

${ }^{*}$ These authors contributed equally to this research.

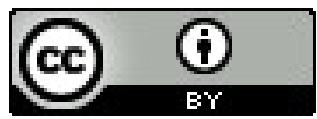

This work is licensed under a Creative Commons Attribution International 4.0 License.

SIGCSE '21, March 13-20, 2021, Virtual Event, USA.

(C) 2021 Copyright held by the owner/author(s).

ACM ISBN 978-1-4503-8062-1/21/03.

https://doi.org/10.1145/3408877.3432561 52nd ACM Technical Symposium on Computer Science Education (SIGCSE '21), March 13-20, 2021, Virtual Event, USA. ACM, New York, NY, USA, 6 pages. https://doi.org/10.1145/3408877.3432561

\section{INTRODUCTION}

The COVID-19 pandemic forced universities across the globe to transition to remote instruction in March of 2020 [7]. While the Spring 2020 offering of our course was able to adapt to some degree, we were not satisfied with the final results due to the abrupt nature of the move.

Fortunately, we were given three months to plan and build a version of the course optimized for remote instruction for Summer 2020. In redesigning the structure of the course, our primary goal was to make it as accessible as possible [5] and to provide a flexible experience for students regardless of where they were situated. This paper details several components of the Summer 2020 offering of our course, of which the first two authors of this paper were instructors.

\section{COURSE OVERVIEW}

Our course, Principles and Techniques of Data Science [2], is a second course in data science at the University of California, Berkeley. In the course, students learn how to use practical data science tools, such as pandas, seaborn, and scikit-learn while simultaneously developing an understanding of the theory behind common techniques in machine learning including linear regression, logistic regression, and gradient descent. Most of the assignments require students to code in Python within the Jupyter notebook environment, but a small portion of assignments are conducted in a traditional pencil and paper format.

In a typical semester the course is co-taught by one faculty member from the Computer Science department and one from the Statistics department. Enrollment exceeded 1000 students per semester in recent fall/spring iterations. Our offering in Summer 2020 was just the second summer offering of the course, and it had an enrollment of over 300 students. For reference, the pilot summer offering had just over 100 students. The scale of the course dictated the need to rely on teaching assistants to teach discussion and lab sections, hold office hours, and help develop content for assignments and exams.

In order to gauge the effectiveness of each of the changes we implemented this summer and to maintain a constant feedback loop between staff and students, we decided to include weekly surveys as a small portion of students' grades. Students received credit by simply submitting surveys on time, and the content of their responses did not affect their grade. Some of these weekly surveys were anonymous to allow students to voice any serious concerns they had with the course. Responses to these surveys allowed us to 
quickly adapt to student concerns, and we were able to make small but significant changes to improve the student experience.

\section{LECTURE FORMAT}

In a typical fall/spring semester our course consists of two 80minute lectures per week. Prior to the switch to remote instruction, lectures were presented live in a large lecture hall, and the lectures were recorded for students who did not attend in person. The Summer 2020 iteration of our course was a condensed 8-week offering, which meant that the number of 80-minute lectures each week had to increase from two to four.

\subsection{Lecture Content}

Instead of delivering four 80-minute lectures per week synchronously, we opted to deliver lecture content asynchronously. In the spring we observed in several courses that attendance at synchronized online lecture sessions was often poor, with students instead opting to watch recordings posted later. Knowing this, we decided to spend our efforts on crafting and curating high quality lectures designed for asynchronous consumption rather than dedicating a large amount of effort to a lecture format that would be underattended and generally inferior to the typical live lecture experience.

Each "lecture" consisted of a playlist of 4 to 7 videos, each between 3 and 15 minutes in length, with a total run-time between 65 and 80 minutes. Based on recommendations [9], we tried to keep video lengths as close to 6 minutes as possible to maximize student attention. However, a natural "split" was sometimes impossible at that mark due to the nature of the presented content. Student feedback was mixed; while many students appreciated that lecture videos were broken into easily-digestible chunks, others indicated that they would have preferred each lecture to be presented as a single 80-minute long video. We hypothesized that students in the latter camp preferred this format because they were used to watching lecture recordings in this manner in a traditional semester.

Roughly half of the video content was recorded by instructors over the summer, with the other half being reused from previous semesters. Reusing videos from previous semesters allowed us to leverage the content experience of the various faculty that teach our course, to provide students with different perspectives on the material, and to redirect our efforts towards other aspects of the course.

However, reusing old videos came with its own challenges. Only a small fraction of videos from previous semesters were recorded specifically for asynchronous consumption; the majority of old content had to be cut from 80-minute long videos recordings of live lectures. Instructors delivering live lectures often did not structure their lessons in a modular fashion, so dividing up this content took great care and effort. Furthermore, many students expressed that they preferred the newly-recorded lectures since they were far more succinct; this is not only because our new videos were devoid of any student dialogue but also because we were able to rerecord each segment until we were satisfied with its clarity and concision. Students also expressed that they preferred to see a talking head of the instructor while watching a video; our newly-recorded videos had these deliberately [9]. By contrast, the in-person lecture recording system at our institution generally only captures the instructor's screen; hence, the old lecture videos we used did not contain a video of the instructor's face. Lastly, some students also noted the lack of cohesion between lectures since many lectures were presented in various different styles. If given more time, we would naturally have recorded more lectures specifically tailored to the asynchronous format to alleviate the aforementioned concerns.

These videos were embedded in a web page that was linked directly on the course website [2]. These web pages also included links to the slides and code that were presented in the videos, along with links to supplemental reading when applicable. Each lecture had its own dedicated thread on Piazza, the online discussion forum used by our course; these threads allowed both students and instructors to ask and answer questions about that lecture's concepts. We released four lectures each week, on the mornings of Monday, Tuesday, Wednesday, and Thursday. We intentionally did not release all lecture content at the start of the week in order to encourage students to create a schedule for themselves to consume lectures.

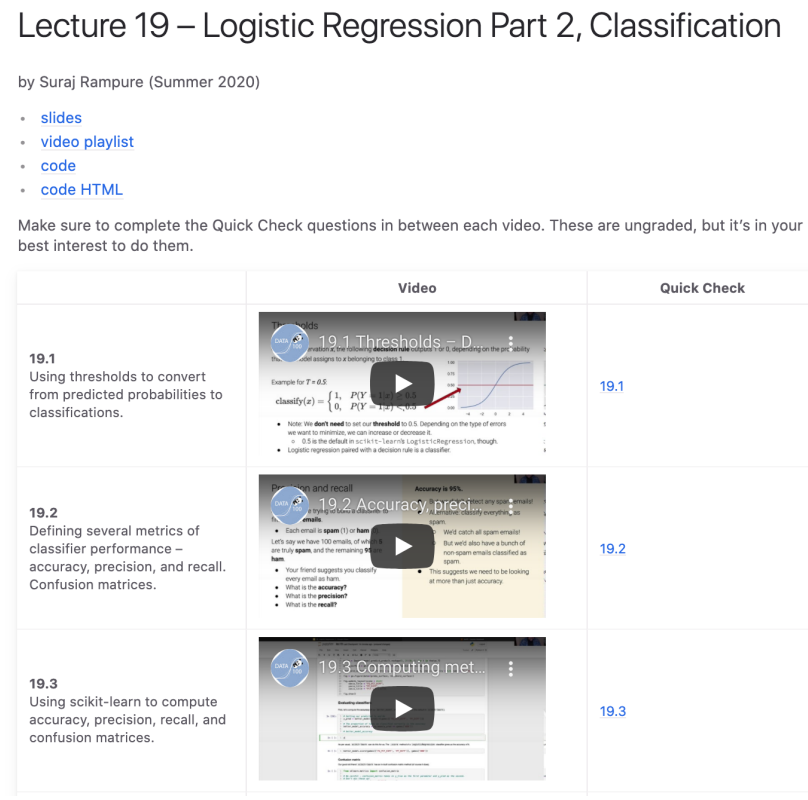

Figure 1: A lecture webpage with embedded videos and links to Quick Checks, slides, and code. [1]

\subsection{Quick Checks}

In between each video we included a link to a "Quick Check" which provided students with an opportunity for immediate practice with the content of the video they had just watched $[6,12]$.

Each Quick Check was a Google Form consisting of one to four questions. These questions were mostly multiple-choice and selectall; however, we also included some short-answer problems that had students answer a mathematical question (for instance, "What is the $L_{2}$ loss of the prediction above?") and even some long-answer problems. Answers were provided to students immediately after submission. Quick Checks were included to motivate students to 
actively process the information they were learning, rather than passively listen to the prerecorded lecture videos [14]. Other studies have shown the effectiveness of multiple-choice Quick Check questions to be limited and instead have encouraged the use of autograded free-response questions [11]; however, we did not reference such studies when designing our course.

Even though Quick Checks were entirely optional, roughly $50 \%$ of the course regularly completed them. Each lecture web page also included a link to a YouTube playlist for students who wanted to watch the videos back-to-back directly on YouTube and to not engage with the Quick Checks.

Based on student feedback, Quick Checks were immensely helpful for establishing a baseline understanding of lecture content. Some students frequently requested more difficult problems (in particular, those from exams in past semesters) to be included as a part of Quick Checks. Furthermore, we initially only included answers to Quick Check problems and not explanations because we did not think they were necessary, but at the request of students we started to include in-depth explanations as well. This signalled to us that students may have referenced Quick Check questions to help them study for exams.

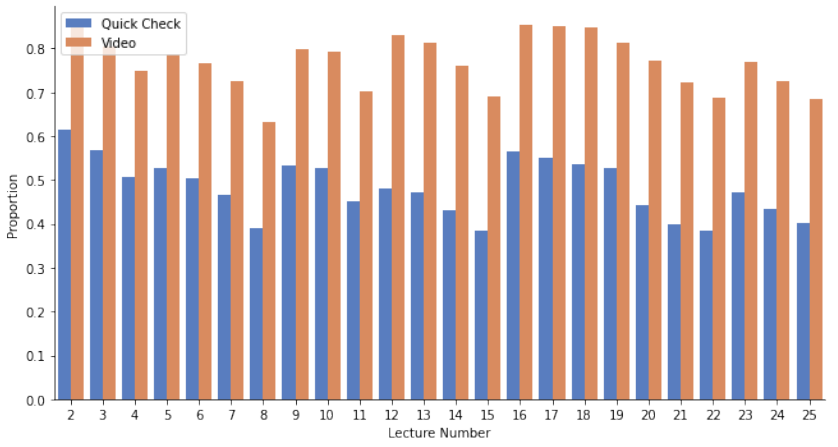

Figure 2: The proportion of students in the class who watched lecture videos and submitted Quick Checks for each lecture.

\subsection{Live Lecture Recaps}

Quick Checks and lecture threads on Piazza served as two avenues for instructors to identify students' misconceptions with lecture concepts. We used this information to plan optional live lecture recap sessions held weekly on Friday using Zoom. Each live lecture recap was meant to summarize and clarify ideas from the previous four days' lectures.

Live lecture recaps were 50 minutes long and were always cotaught by both of the instructors. The instructors would take 15 minute rotations in presenting concepts, and the instructor not actively lecturing would intentionally interject with relevant commentary or alternative explanations of ideas. This was to foster a more casual, conversational learning environment for students [15] rather than to serve as another monologue-like lecture since they already had four lectures each week.

In addition to serving as an opportunity to strengthen students' understanding of lecture concepts, live lecture recaps also acted as a forum for student-instructor interaction, which was largely reduced by the decision to present lectures asynchronously. On average, roughly $10 \%$ of the class attended these sessions; for comparison, this is around the same as the proportion of students who attended live lectures in previous physical semesters of this course. Since these sessions were recorded and posted for students who did not attend, it is likely that only the students who valued the ability to ask instructors questions directly chose to attend these sessions. Anecdotally, the students who attended these sessions were the most active on Piazza and regularly attended the instructors' office hours for conceptual help.

It's important to note here that our course was not flipped. In flipped courses $[3,13]$, synchronous lecture components are usually more frequent and more integral to the student experience. In our course, synchronous lectures were purely supplementary and not focused on problem-solving, and many students did well without ever attending them.

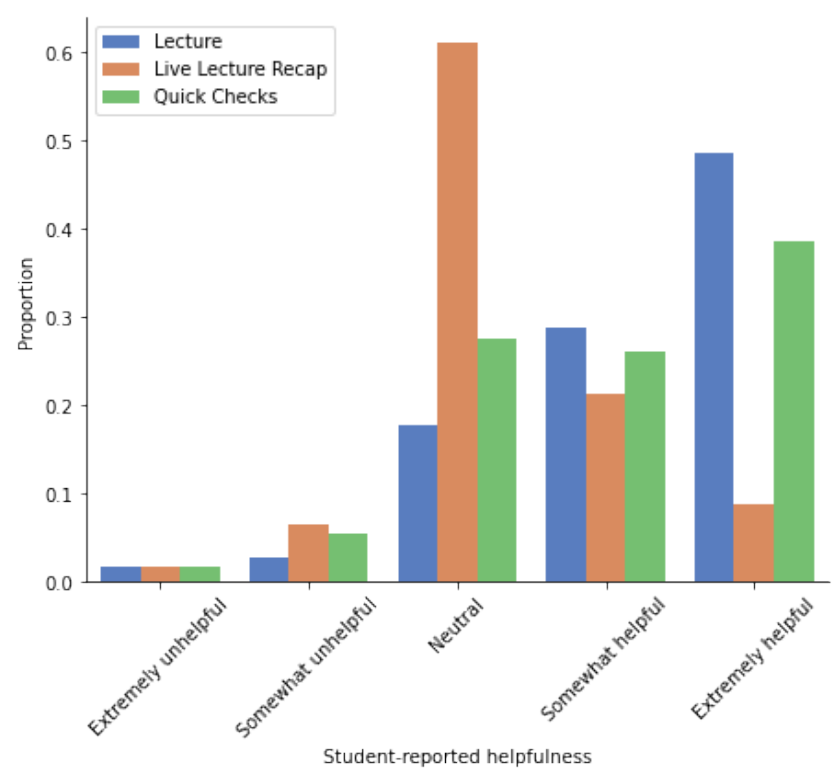

Figure 3: The helpfulness of Lectures, Live Lecture Recaps, and Quick Checks, as self-reported by students in an end-ofsemester survey.

\section{SUPPLEMENTAL SECTIONS}

The course has a variety of sections that complement lectures. Drop-in office hours were hosted for several hours each day by teaching assistants and tutors; students would access these office hours by creating a ticket on a website that featured an office hours queue. Although some students came to office hours with conceptual questions, most students attended office hours to receive help with assignments. In addition to office hours, the course featured discussion and lab sections. 


\subsection{Discussion Sections}

Traditionally, 50-minute discussion sections were offered every week for a small group of around 30 students. These discussion sections, which were taught by teaching assistants, were intended to supplement the material presented in lecture. For the more practical concepts in the course, discussion sections were accompanied by a Jupyter notebook consisting of short coding exercises for students to work on. For the more theoretical concepts in the course, discussion sections were accompanied by a worksheet which consisted of several practice problems on the relevant concepts. For the condensed summer offering of the course, there were two discussion sections per week.

We gave students the flexibility to consume discussion content in two different ways. The first way was to watch prerecorded discussion videos, and the second way was to attend a live discussion section. For each discussion, a set of teaching assistants created a playlist of videos that walked through all of the problems on the discussion worksheet while another set of teaching assistants held live discussion sections at various times of the day. We found that around $40 \%$ of students watched the prerecorded discussion videos while only around $10 \%$ of students attended live discussions.

Initially, we provided a live "discussion recap" section that students could attend after watching prerecorded discussion videos so that they could ask additional questions. Only 3\% of the class regularly attended discussion recap sections, likely because students could easily just attend drop-in office hours instead. As a result, we cancelled them after the 3rd week.

To replace the discussion recap sections, we introduced synchronous "exam prep" sections after the 3rd week. In these exam prep sections, a teaching assistant would go over a few past exam problems related to the topics in the corresponding discussion section. Past exam problems were generally more challenging than the problems presented in discussion worksheets, so it was helpful for students to see a teaching assistant break down complex problems into simpler subparts. During exam weeks, around 15\% of the class attended one of these exam prep sections while only around $10 \%$ of the class attended in non-exam weeks.

\subsection{Lab Format}

In fall/spring semesters, students in our course are given a single lab assignment each week; in the summer this was doubled to two lab assignments per week. Traditionally, lab sections were offered in person for groups of around 30 students; students could come to lab sections to work with other students on the lab assignment. Most teaching assistants ran lab sections similar to office hours; they would be available to answer any questions related to the lab, but they would not spend time presenting material to the entire class.

Once the COVID-19 pandemic hit, the Spring 2020 offering of our course abolished labs completely. Students were instead encouraged to go to drop-in office hours if they had questions about the lab assignment. While this provided students with a way to receive help on the lab assignment, we felt that this format deprived students of the collaborative format of in-person lab sections.
As a result, we introduced remote lab sections for the first time this summer. During these synchronous lab sections, teaching assistants would walk through portions of the lab assignment. The primary goal of these live lab sections was to help students understand the deeper data science concepts behind the lab assignments, rather than to encourage them to simply write code that passed the autograder tests. Live lab sections were by far the most popular synchronous event in the course, with roughly $25 \%$ of the class attending these biweekly sections. However, some students admitted afterwards that they only attended these sections because solutions to the lab were being given out.

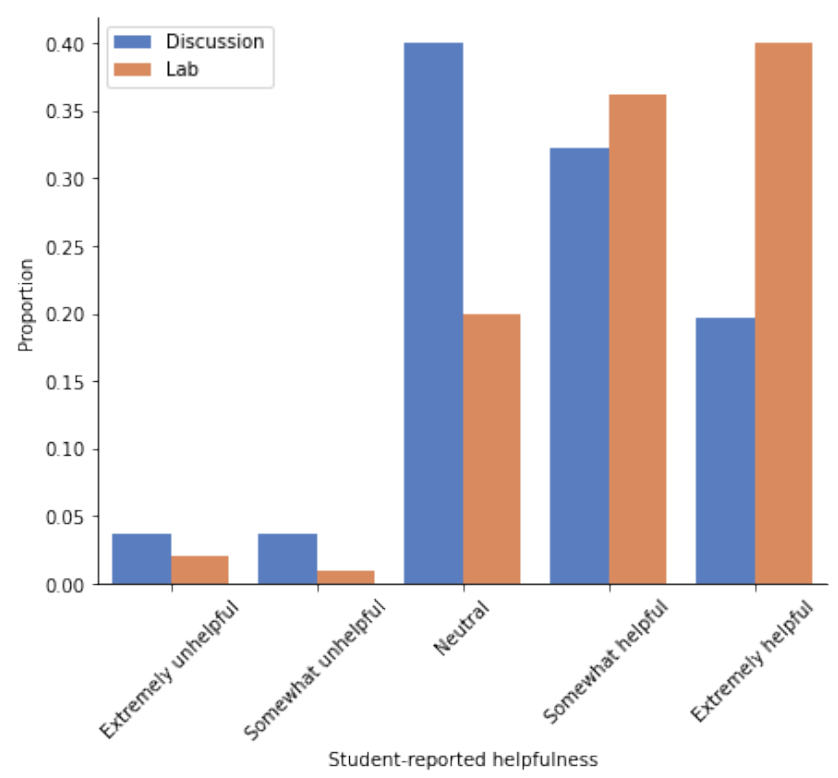

Figure 4: The helpfulness of Discussions and Labs, as selfreported by students in an end-of-semester survey.

\section{EXAMINATIONS}

In the in-person setting most courses in our department administered exams in person and on paper; our course was no exception. Because of the pandemic, the Spring 2020 offering simply had no exams, so we had no in-house precedent for an exam format to use. As such, we decided to experiment with both web-based and on-paper exams [8], depending on the content that was in scope for the exam.

Our offering of the course consisted of two midterm exams, which were administered at the end of the third week and the start of the sixth week respectively, and a final exam administered at the end of the semester. As is detailed below, our final examination was administered over the course of two days as two separate oneday exams. Regardless of the format, each exam was taken by all students at the same time (with the minor exception of students in time zones far from ours, for whom we provided alternate exams). Each exam lasted 1 hour and 30 minutes and was worth the same weight in students' grades. 


\subsection{Web-Based Exams}

The first third of our course focuses on teaching students how to use practical tools in data science. As such, the content of the first midterm tends to involve pandas and SQL coding questions, as well as multiple-choice questions about visualizations and the data science life cycle (a guiding pedagogical tool in our course). It is wellunderstood $[4,10]$ that programming exams that ask students to write code on a computer are more accurate assessments of students' abilities to program than exams on paper are. Furthermore, we wanted to offer exams in a format that would support randomization and versioning in order to mitigate collaboration since we made the decision not to employ remote proctoring tools. For these reasons, we opted to use a web-based tool for our first midterm.

Gradescope, the submission platform for assignments in our course, provides the ability to administer web-based exams with both multiple-choice and free-response questions. However, it is limited in that it does not directly support randomization or versioning. We instead used an open-source tool built by teaching assistants for another course in our department; this tool provided similar functionality to Gradescope but with randomization and versioning. As the tool was brand-new, we deployed a "practice exam" to students in order for them to gain familiarity with the environment before the day of the official exam. The tool allowed us to deploy a slightly different version of the exam to each student.

- Randomization. Each student received the same problems but in a different order. For multiple-choice questions whose answer choices had no inherent ordering, students also received shuffled answer choices for each problem.

- Versioning. For certain questions on the exam, we created multiple versions that were slightly different from one another. For questions that asked students to write freeresponse code, we changed the prompts ever-so-slightly. For instance, one problem asked students to determine the number of video game consoles sold by Microsoft; other versions of the problem asked students to find that number for Sega and Nintendo instead.

Versioning above allowed us to detect students who potentially collaborated with others because some students had final answers that did not correspond to their question versions. To prove these suspicions, we used the tool's logging system, which saved the state of students' exams at regular intervals. This made it easy to tell when students copied in answers from elsewhere.

\subsection{Paper-Based Exams}

The latter two-thirds of our course focuses on the theory behind classical machine learning algorithms. Historically, the second midterm for the course tends to ask many questions involving algebra and derivations (e.g. "What is the guess for $\hat{\theta}$ after one iteration of gradient descent? Show your work."). The web-based tool used for the first midterm would not be appropriate for such content; thus, we decided to use a more traditional paper-based exam for the second midterm.

Similar to the first midterm, the exam was time-boxed. Students had an hour and forty-five minutes (with the extra fifteen minutes dedicated to the scanning process) to download the exam PDF from
Gradescope, write their answers on blank paper, scan their answers, and turn in their scanned answers on Gradescope.

While the on-paper format was better suited this content, we made the mistake of including too many multiple-choice problems on the second midterm, which diminished the value of this format. Students did not generally have any objections to the fact that the midterm exams were in different formats.

\subsection{Final Exam}

The final exam was cumulative, and we wanted to assess all content in the course in a way that best suited that content. Fortunately, summer courses at our institution are not bound by campus rules which typically dictate a specific three-hour window during which the final exam must take place, so we were able to offer the final over a two-day period.

The first day of the exam was administered using the tool used for the first midterm. It included free-response coding questions as well as multiple-choice questions. The second day of the exam was administered on-paper, similar to the second midterm, but this time the exam included no multiple-choice questions.

The motivating factor behind our decision to administer the exam as two separate hour-and-a-half long exams on consecutive days rather than as a single three-hour long exam was our desire to reduce the stakes of the final exam. In theory, one bad day would not ruin a student's grade in the course. However, the two-day format was not well-received by students. On a post-mortem survey, many students expressed the sentiment that this format caused more stress instead of less since they had to worry about the act of writing the exam over the course of 27 hours instead of just 3 hours. Students also noted that the content was not delimited between the two days (i.e. content from all parts of the course appeared on both days of the exam); they expressed dissatisfaction that they were unable to target their studying towards specific topics on each day.

\section{CONCLUSION AND KEY TAKEAWAYS}

Given several months to plan an entirely remote offering of an existing upper-division data science course, we were able to adhere to our goal of offering as flexible of an experience as possible for students. We evidently did not get everything perfect from the beginning, but weekly survey feedback from students allowed us to constantly improve the course to meet student needs. By the end of the summer, we were able to offer a version of the course that satisfied both the staff and students.

It is worth noting that roughly $70 \%$ of the students in our course did not attend any synchronous components (lecture recaps, discussion sections, and lab sections). This corroborates the observations that instructors of other similarly-run courses during our semester made. If we were to teach this class remotely again, we would do a more thorough analysis of flipped classroom literature to identify synchronous lecture formats that may lead to improved attendance and educational attainment, if any.

Our most significant takeaways from our experiences this summer are presented below.

- While students may make complaints about the lack of consistency, a course can still function well with lecture videos that feature multiple instructors and multiple formats. 
- In a course where lecture is primarily asynchronous, conceptual questions that supplement lecture videos are useful in giving students immediate feedback on their understanding of lecture concepts, and having an official exercise to complete encourages students to stay on task. Students completed these activities even though they were not required We recommend providing such questions after every short lecture video.

- When stuck with online discussion sections, our students mostly preferred prerecorded videos that walked through the discussion worksheet, although a small fraction of students preferred a more typical synchronous format. For the purposes of flexibility, we would recommend offering both asynchronous and synchronous formats for discussion sections.

- For lab sections, we would recommend striking a balance between giving away approaches to problems and giving away solutions. When given solutions, students often copy them exactly without understanding the underlying concepts. One potential idea would be for teaching assistants to walk through problems similar to (but not exactly the same as) the ones in the lab assignment. This would force students to understand the concepts behind the particular problem instead of simply copying the problem's solutions.

- Do not be afraid to employ a variety of radically different exam formats.

- Regularly survey your students. Students were happy to provide feedback even on a weekly basis. Feedback was not only immeasurably helpful for staff, but also it gave students the feeling that they had some ownership in the operation of the course and that the instructors truly cared about their thoughts.

\section{ACKNOWLEDGMENTS}

We would like to acknowledge the efforts of our teaching staff and in particular our teaching assistants, without whom this course would not have been possible. We would also like to acknowledge the numerous faculty members with whom we consulted before and during the course for their valuable input as to how to navigate such an unprecedented semester.

\section{REFERENCES}

[1] 2020. Lecture 19 - Logistic Regression Part 2, Classification. Retrieved November 23rd, 2020 from http://ds100.org/su20/lecture/lec19/

[2] 2020. Principles and Techniques of Data Science. Retrieved November 23rd, 2020 from http://ds100.org/su20/

[3] Ashish Amresh, Adam Carberry, and John Femiani. 2013. Evaluating the effectiveness of flipped classrooms for teaching CS1. Proceedings - Frontiers in Education Conference, 733-735. https://doi.org/10.1109/FIE.2013.6684923

[4] Mary Elaine Califf and Mary Goodwin. 2002. Testing Skills and Knowledge: Introducing a Laboratory Exam in CS1. In Proceedings of the 33rd SIGCSE Technical Symposium on Computer Science Education (SIGCSE '02). Association for Computing Machinery, New York, NY, USA, 217-221. https://doi.org/10.1145/563340.563425

[5] Nicholas Casey. 2020. College Made Them Feel Equal. The Virus Exposed How Unequal Their Lives Are. Retrieved August 27th, 2020 from https://www.nytimes. com/2020/04/04/us/politics/coronavirus-zoom-college-classes.html

[6] Quintin Cutts, Angela Carbone, and Kelsey van Haaster. 2004. Using an electronic voting system to promote active reflection on coursework feedback. In Proceedings of Intl. Conf. on Computers in Education, Melbourne, Australia.

[7] Chris Boyette Eric Levenson and Janine Mack. 2020. Colleges and universities across the US are canceling in-person classes due to coronavirus. Retrieved August 25th, 2020 from https://www.cnn.com/2020/03/09/us/coronavirus-universitycollege-classes/index.html

[8] Edward Gehringer. 2010. Online Vs. On Paper Exams. In 2010 Annual Conference \& Exposition. ASEE Conferences, Louisville, Kentucky. https://peer.asee.org/16349.

[9] Philip J. Guo, Juho Kim, and Rob Rubin. 2014. How Video Production Affects Student Engagement: An Empirical Study of MOOC Videos. In Proceedings of the First ACM Conference on Learning @ Scale Conference (L@S '14). Association for Computing Machinery, New York, NY, USA, 41-50. https://doi.org/10.1145/ 2556325.2566239

[10] Oka Kurniawan, Norman Tiong Seng Lee, and Christopher M. Poskitt. 2020. Securing Bring-Your-Own-Device (BYOD) Programming Exams. In Proceedings of the 51st ACM Technical Symposium on Computer Science Education (SIGCSE '20). Association for Computing Machinery, New York, NY, USA, 880-886. https: //doi.org/10.1145/3328778.3366907

[11] Lisa L. Lacher, Albert Jiang, Yu Zhang, and Mark C. Lewis. 2018. Including Coding Questions in Video Quizzes for a Flipped CS1. In Proceedings of the 49th ACM Technical Symposium on Computer Science Education (SIGCSE '18). Association for Computing Machinery, New York, NY, USA, 574-579. https: //doi.org/10.1145/3159450.3159504

[12] Roberto E. Lopez-Herrejon and Morrie Schulman. 2004. Using Interactive Technology in a Short Java Course: An Experience Report. In Proceedings of the 9th Annual SIGCSE Conference on Innovation and Technology in Computer Science Education (ITiCSE '04). Association for Computing Machinery, New York, NY, USA, 203-207. https://doi.org/10.1145/1007996.1008051

[13] Greg Mason, Teodora Rutar Shuman, and K.E. Cook. 2013. Comparing the Effectiveness of an Inverted Classroom to a Traditional Classroom in an UpperDivision Engineering Course. Education, IEEE Transactions on 56 (11 2013), 430-435. https://doi.org/10.1109/TE.2013.2249066

[14] Jeffrey J. McConnell. 1996. Active Learning and Its Use in Computer Science. In Proceedings of the 1st Conference on Integrating Technology into Computer Science Education (ITiCSE '96). Association for Computing Machinery, New York, NY, USA, 52-54. https://doi.org/10.1145/237466.237526

[15] William Waite, Michele Jackson, and Amer Diwan. 2003. The Conversational Classroom. ACM SIGCSE Bulletin 35 (02 2003). https://doi.org/10.1145/611892. 611950 